\title{
Erratum to: Stability of Diffusion Coefficients in an Inverse Problem for the Lotka-Volterra Competition System
}

\author{
K. Sakthivel • N. Baranibalan · J.-H. Kim • \\ K. Balachandran
}

Published online: 19 June 2010

(C) Springer Science+Business Media B.V. 2010

\section{Erratum to: Acta Appl Math \\ DOI 10.1007/s10440-009-9455-z}

The construction of the weight function $\psi(x)$ in (2.1) and Assumption 3.1 are inconsistent. Therefore Assumption 3.1 on the weight function $\psi(x)$ should be corrected as follows:

Assumption 3.1 Suppose $A_{i}^{\theta} \in C^{3}(\Omega), i=1,2$ be a real valued function such that $A_{i}^{\theta}, i=1,2$ and all its derivatives up to order three are bounded and also it satisfies $\left|\nabla \psi \cdot \nabla A_{i}^{\theta}\right| \geq \sigma>0, i=1,2$ on $\overline{\Omega \backslash \omega_{0}}$, where $\omega_{0} \Subset \omega \Subset \Omega$.

This assumption does not change the results in Sect. 2. In Sect. 3, the statement after (3.1), "Let us remark that ... arguments in [19]." should be removed and Lemma 3.1 should be replaced by the following

Lemma 3.1 Consider the first order partial differential operator $P_{0} h=\nabla A^{\theta} \cdot \nabla h$, where $A^{\theta}$ satisfies Assumption 3.1. Then there exists a constant $C>0$, such that for sufficiently large $\lambda$ and $s$, the following inequality holds:

$$
s^{2} \lambda^{2} \int_{\Omega} \phi^{\theta} e^{-2 s \alpha^{\theta}}|h|^{2} d x \leq C\left(\int_{\Omega} \frac{1}{\phi^{\theta}} e^{-2 s \alpha^{\theta}}\left|P_{0} h\right|^{2} d x+s^{2} \lambda^{2} \int_{\omega_{0}} \phi^{\theta} e^{-2 s \alpha^{\theta}}|h|^{2} d x\right),
$$

with $\theta \in(0, T)$ and for $h \in H_{0}^{1}(\Omega)$.

The online version of the original article can be found under doi:10.1007/s10440-009-9455-z.

K. Sakthivel (凶) · J.-H. Kim

Department of Mathematics, Yonsei University, 120749 Seoul, Korea

e-mail: pktsakthi@gmail.com

N. Baranibalan $\cdot$ K. Balachandran

Department of Mathematics, Bharathiar University, 641046 Coimbatore, India 
Proof Let us consider $v=e^{-s \alpha^{\theta}} h$ and so $Q_{0} v=e^{-s \alpha^{\theta}} P_{0}\left(e^{s \alpha^{\theta}} v\right)=s v P_{0} \alpha^{\theta}+P_{0} v$, where we note that $h \in H_{0}^{1}(\Omega)$. Then by integration by parts with respective to space variable, we obtain

$$
\begin{aligned}
\int_{\Omega} \frac{1}{\phi^{\theta}}\left(Q_{0} v\right)^{2} d x= & s^{2} \int_{\Omega} \frac{1}{\phi^{\theta}}\left(P_{0} \alpha^{\theta}\right)^{2} v^{2} d x+\int_{\Omega} \frac{1}{\phi^{\theta}}\left(P_{0} v\right)^{2} d x+2 s \int_{\Omega} \frac{1}{\phi^{\theta}} v\left(P_{0} \alpha^{\theta}\right)\left(P_{0} v\right) d x \\
= & s^{2} \lambda^{2} \int_{\Omega} \phi^{\theta}\left(\nabla A^{\theta} \cdot \nabla \psi\right)^{2} v^{2} d x+\int_{\Omega} \frac{1}{\phi^{\theta}}\left(P_{0} v\right)^{2} d x \\
& -2 s \lambda \int_{\Omega}\left(\nabla A^{\theta} \cdot \nabla \psi\right)\left(\nabla A^{\theta} \cdot \nabla v\right) v d x \\
\geq & s^{2} \lambda^{2} \int_{\Omega} \phi^{\theta}\left|\nabla \psi \cdot \nabla A^{\theta}\right|^{2}|v|^{2} d x+s \lambda \int_{\Omega} \nabla\left(P_{0} \psi \nabla A^{\theta}\right)|v|^{2} d x .
\end{aligned}
$$

Using Assumption 3.1, one can get

$$
s^{2} \lambda^{2} \int_{\Omega} \phi^{\theta}\left|\nabla \psi \cdot \nabla A^{\theta}\right|^{2}|v|^{2} d x \geq \sigma^{2} s^{2} \lambda^{2}\left(\int_{\Omega} \phi^{\theta}|v|^{2} d x-\int_{\omega_{0}} \phi^{\theta}|v|^{2} d x\right)
$$

and hence

$$
s^{2} \lambda^{2} \sigma^{2}\left(\int_{\Omega} \phi^{\theta}|v|^{2} d x-\int_{\omega_{0}} \phi^{\theta}|v|^{2} d x\right) \leq \int_{\Omega} \frac{1}{\phi^{\theta}}\left|Q_{0} v\right|^{2} d x+s \lambda \int_{\Omega}\left|\nabla\left(P_{0} \psi \nabla A^{\theta}\right)\right||v|^{2} d x .
$$

Again from Assumption 3.2, we have

$$
\begin{aligned}
s^{2} \lambda^{2} \sigma^{2} \int_{\Omega} e^{-2 s \alpha^{\theta}} \phi^{\theta}|h|^{2} d x \leq & c_{1} T^{2} s \lambda \int_{\Omega} e^{-2 s \alpha^{\theta}} \phi^{\theta}|h|^{2} d x+\int_{\Omega} e^{-2 s \alpha^{\theta}} \frac{1}{\phi^{\theta}}\left|P_{0} h\right|^{2} d x \\
& +s^{2} \lambda^{2} \sigma^{2} \int_{\omega_{0}} e^{-2 s \alpha^{\theta}} \phi^{\theta}|h|^{2} d x .
\end{aligned}
$$

Finally, taking $\lambda \geq 1, s \geq 2 c_{1} T^{2} / \sigma^{2}$ one can conclude the lemma.

The Proposition 3.1 also needs some minor revision.

Proposition 3.1 Let $\left(B_{1}, B_{2}, B_{3}\right)$ be the solutions of (1.3) and $u, v, w$ be the solutions of (3.1). Suppose all the conditions of Theorem 2.1 and Assumption 3.1 are satisfied. Then there exists a constant $C\left(\gamma_{1}, \gamma_{2}, \delta, \sigma\right)>0$, such that for sufficiently large $s$ and $\lambda$, the following estimate holds:

$$
\begin{aligned}
& s^{2} \lambda^{2} \int_{\Omega} \phi^{\theta} e^{-2 s \alpha^{\theta}}\left(|f|^{2}+|g|^{2}+|\nabla f|^{2}+|\nabla g|^{2}\right) d x \\
& \quad \leq C\left(\gamma_{1}, \gamma_{2}, \delta, \sigma\right) \sum_{i=1}^{7} \mathcal{E}_{i}(\theta)+C s^{2} \lambda^{2} \int_{\omega_{0}} \phi^{\theta} e^{-2 s \alpha^{\theta}}\left(|f|^{2}+|g|^{2}+|\nabla f|^{2}+|\nabla g|^{2}\right) d x,
\end{aligned}
$$

for any $f, g \in H_{0}^{2}(\Omega)$, where the functions $\mathcal{E}_{i}, i=1, \ldots, 7$ are given in (3.2).

Proof From the value of the solutions satisfying (3.1a) at $t=\theta$ and the definition of $F$, we arrive at

$$
P_{0} f=\nabla \tilde{A}_{1}^{\theta} \cdot \nabla f=u^{\theta}-\nabla\left(d_{1} \nabla B_{1}^{\theta}\right)+a_{1}^{\theta} B_{1}^{\theta}+a_{2}^{\theta} B_{2}^{\theta}+a_{3}^{\theta} B_{3}^{\theta}-f \Delta \widetilde{A}_{1}^{\theta},
$$


and therefore, using Lemma 3.1, we obtain

$$
\begin{aligned}
s^{2} \lambda^{2} \int_{\Omega} \phi^{\theta} e^{-2 s \alpha^{\theta}}|f|^{2} d x \leq & C\left(\int_{\Omega} \frac{1}{\phi^{\theta}} e^{-2 s \alpha^{\theta}}\left|P_{0} f\right|^{2} d x+s^{2} \lambda^{2} \int_{\omega_{0}} \phi^{\theta} e^{-2 s \alpha^{\theta}}|f|^{2} d x\right) \\
\leq & C \int_{\Omega} \frac{1}{\phi^{\theta}} e^{-2 s \alpha^{\theta}}\left(\left|u^{\theta}\right|^{2}+\left|\nabla d_{1}\right|^{2}\left|\nabla B_{1}^{\theta}\right|^{2}+\left|d_{1}\right|^{2}\left|\Delta B_{1}^{\theta}\right|^{2}\right. \\
& \left.+\left|a_{1}^{\theta}\right|^{2}\left|B_{1}^{\theta}\right|^{2}+\left|a_{2}^{\theta}\right|^{2}\left|B_{2}^{\theta}\right|^{2}+\left|a_{3}^{\theta}\right|^{2}\left|B_{3}^{\theta}\right|^{2}+\left|f \Delta \widetilde{A}_{1}^{\theta}\right|^{2}\right) d x \\
& +C s^{2} \lambda^{2} \int_{\omega_{0}} \phi^{\theta} e^{-2 s \alpha^{\theta}}|f|^{2} d x \\
\leq & C\left(\gamma_{1}, \delta\right)\left(\mathcal{E}_{1}(\theta)+\mathcal{E}_{5}(\theta)\right)+C s^{2} \lambda^{2} \int_{\omega_{0}} \phi^{\theta} e^{-2 s \alpha^{\theta}}|f|^{2} d x
\end{aligned}
$$

From the solutions satisfying (3.1b) at $t=\theta$ and the definition of $G$, we have

$$
P_{0} g=\nabla \widetilde{A}_{2}^{\theta} \cdot \nabla g=v^{\theta}-\nabla\left(d_{2} \nabla B_{2}^{\theta}\right)+a_{4}^{\theta} B_{2}^{\theta}+a_{5}^{\theta} B_{3}^{\theta}+a_{6}^{\theta} B_{1}^{\theta}-g \Delta \widetilde{A}_{2}^{\theta}
$$

whence it follows that

$$
\begin{aligned}
s^{2} \lambda^{2} \int_{\Omega} \phi^{\theta} e^{-2 s \alpha^{\theta}}|g|^{2} d x \leq & C\left(\int_{\Omega} \frac{1}{\phi^{\theta}} e^{-2 s \alpha^{\theta}}\left|P_{0} g\right|^{2} d x+s^{2} \lambda^{2} \int_{\omega_{0}} \phi^{\theta} e^{-2 s \alpha^{\theta}}|g|^{2} d x\right) \\
\leq & C \int_{\Omega} \frac{1}{\phi^{\theta}} e^{-2 s \alpha^{\theta}}\left(\left|v^{\theta}\right|^{2}+\left|\nabla d_{2}\right|^{2}\left|\nabla B_{2}^{\theta}\right|^{2}+\left|d_{2}\right|^{2}\left|\Delta B_{2}^{\theta}\right|^{2}\right. \\
& \left.+\left|a_{4}^{\theta}\right|^{2}\left|B_{2}^{\theta}\right|^{2}+\left|a_{5}^{\theta}\right|^{2}\left|B_{3}^{\theta}\right|^{2}+\left|a_{6}^{\theta}\right|^{2}\left|B_{1}^{\theta}\right|^{2}+\left|g \Delta \tilde{A}_{2}^{\theta}\right|^{2}\right) d x \\
& +C s^{2} \lambda^{2} \int_{\omega_{0}} \phi^{\theta} e^{-2 s \alpha^{\theta}}|g|^{2} d x \\
\leq & C\left(\gamma_{2}, \delta\right)\left(\mathcal{E}_{2}(\theta)+\mathcal{E}_{6}(\theta)\right)+C s^{2} \lambda^{2} \int_{\omega_{0}} \phi^{\theta} e^{-2 s \alpha^{\theta}}|g|^{2} d x
\end{aligned}
$$

On the other hand, it is easy to see from the expression $P_{0} f$ that

$$
P_{0} \nabla f=\nabla u^{\theta}-\Delta\left(d_{1} \nabla B_{1}^{\theta}\right)+\nabla\left(a_{1}^{\theta} B_{1}^{\theta}\right)+\nabla\left(a_{2}^{\theta} B_{2}^{\theta}\right)+\nabla\left(a_{3}^{\theta} B_{3}^{\theta}\right)-\nabla\left(f \Delta \widetilde{A}_{1}^{\theta}\right)-\nabla f \Delta \widetilde{A}_{1}^{\theta}
$$

and similarly, we also have

$$
P_{0} \nabla g=\nabla v^{\theta}-\Delta\left(d_{2} \nabla B_{2}^{\theta}\right)+\nabla\left(a_{6}^{\theta} B_{1}^{\theta}\right)+\nabla\left(a_{4}^{\theta} B_{2}^{\theta}\right)+\nabla\left(a_{5}^{\theta} B_{3}^{\theta}\right)-\nabla\left(g \Delta \widetilde{A}_{2}^{\theta}\right)-\nabla g \Delta \widetilde{A}_{2}^{\theta} .
$$

Thus it follows again, from Lemma 3.1 and the computations similar to preceding estimates, that

$$
\begin{aligned}
s^{2} \lambda^{2} \int_{\Omega} \phi^{\theta} e^{-2 s \alpha^{\theta}}\left(|\nabla f|^{2}+|\nabla g|^{2}\right) d x \leq & C\left(\gamma_{1}, \gamma_{2}, \delta\right)\left(\mathcal{E}_{3}(\theta)+\mathcal{E}_{4}(\theta)+\mathcal{E}_{7}(\theta)\right) \\
& +C\left(s^{2} \lambda^{2} \int_{\omega_{0}} \phi^{\theta} e^{-2 s \alpha^{\theta}}\left(|\nabla f|^{2}+|\nabla g|^{2}\right) d x\right) .
\end{aligned}
$$

The proof is thus concluded by combining the above three estimates. 
One can easily see that the proofs of Lemma 3.2 and Lemma 3.3 are not affected by the new Assumption 3.1. Finally the main result stated in Theorem 3.1 should read as follows

Theorem 3.1 Let $\left(B_{1}, B_{2}, B_{3}\right)$ be the solutions of (1.3). Suppose all the assumptions of Theorem 2.1 hold true and $f, g \in H_{0}^{2}(\Omega)$. In addition, suppose that Assumptions 3.1 and 3.2 are also satisfied. Then there exists a constant $C$ with $C\left(\Omega, \omega, T, a_{0}, \delta, \mu_{1}, \mu_{2}, \mu_{3}, \gamma_{1}, \gamma_{2}, \sigma\right)>$ 0 , such that for sufficiently large $\lambda \geq \lambda_{1}$ and $s \geq s_{4}$, the following estimate holds:

$$
\begin{aligned}
& \int_{\Omega}\left(|f|^{2}+|g|^{2}+|\nabla f|^{2}+|\nabla g|^{2}\right) d x \\
& \leq C \int_{\omega_{0}}\left(|f|^{2}+|g|^{2}+|\nabla f|^{2}+|\nabla g|^{2}\right) d x+C\left(\int_{Q_{\omega}}\left(\left|\partial_{t} B_{2}\right|^{2}+\left|\partial_{t} B_{3}\right|^{2}\right) d t d x\right. \\
& \left.\quad+\int_{\Omega}\left(\sum_{i=1}^{3}\left(\left|B_{i}^{\theta}\right|^{2}+\left|\nabla B_{i}^{\theta}\right|^{2}\right)+\sum_{i=1}^{2}\left(\left|\Delta B_{i}^{\theta}\right|^{2}+\left|\nabla\left(\Delta B_{i}^{\theta}\right)\right|^{2}\right)\right) d x\right) .
\end{aligned}
$$

It is easy to see that the proof of Theorem 3.1 remains as such except the last integral term in Proposition 3.1, that is, $s^{2} \lambda^{2} \int_{\omega_{0}} \phi^{\theta} e^{-2 s \alpha^{\theta}}\left(|f|^{2}+|g|^{2}+|\nabla f|^{2}+|\nabla g|^{2}\right) d x$ should be added on the right hand side of four main estimates in the proof.

Moreover, the correction in the main estimate of Theorem 3.1 should be carried out for the statement of the stability result given in (1.4). 